\title{
Nonlinear balance and exchange of stability in dynamics of solitons, peakons, ramps/cliffs and leftons in a $1+1$ nonlinear evolutionary pde
}

\author{
Darryl D. Holm ${ }^{1,2}$ and Martin F. Staley ${ }^{1}$ \\ ${ }^{1}$ Theoretical Division and Center for Nonlinear Studies \\ Los Alamos National Laboratory, MS B284 \\ Los Alamos, NM 87545 \\ email: dholm@lanl.gov, mstaley@lanl.gov \\ ${ }^{2}$ Mathematics Department \\ Imperial College of Science, Technology and Medicine \\ London SW7 2AZ, UK \\ BI3692 \\ ACCEPTED FOR Phys. Lett. A \\ January 16, 2003

\begin{abstract}
We study exchange of stability in the dynamics of solitary wave solutions under changes in the nonlinear balance in a $1+1$ evolutionary partial differential equation related both to shallow water waves and to turbulence. We find that solutions of the equation $m_{t}+u m_{x}+b u_{x} m=\nu m_{x x}$ with $m=u-\alpha^{2} u_{x x}$ for fluid velocity $u(x, t)$ change their behavior at the special values $b=0, \pm 1, \pm 2, \pm 3$.
\end{abstract}

PACS numbers: 11.10.Lm, 03.50.-z, 05.45.Yv, 05.45.-a

Keywords: solitary waves, solitons, peakons, nonlinear evolution, turbulence modeling 
D. D. Holm \& M. F. Staley Solitons, peakons, ramps/cliffs and leftons 2

\section{Contents}

1 Introduction $\quad 2$

1.1 Linear and nonlinear balances in shallow water waves . . . . 2

1.2 Problem statement for the present work . . . . . . . . . 5

$\begin{array}{lll}2 & \text { Exchange of stabilitv } & 6\end{array}$

2.1 Numerics for exchange of stability from $b=0$ to $b= \pm 2 \ldots 6$

2.2 Analytical discussion of the exchange of stability . . . . . . 9

2.3 Confirmations of our numerical methods . . . . . . . . . . . 10

3 Conclusions and further comments 11

\section{Introduction}

\subsection{Linear and nonlinear balances in shallow water waves}

The primary physical mechanism for the propagation of solitary shallow water waves is the balance between nonlinear steepening and linear dispersion. This balance appears uniquely at linear order in an asymptotic expansion in the Korteweg-de Vries equation (KdV),

$$
u_{t}+c_{0} u_{x}+\frac{3 \epsilon_{1}}{2} u u_{x}+\frac{3 \epsilon_{2}}{20} u_{x x x}=0 .
$$

Here the expansion parameters satisfy $\epsilon_{1} \geq \epsilon_{2}>\epsilon_{1}^{2}$ and are defined by $\epsilon_{1}=$ $a / h$ and $\epsilon_{2}=h^{2} / l^{2}$, in terms of $a, h$ and $l$, which denote wave amplitude, mean water depth, and typical horizontal length scale (e.g., a wavelength), respectively. $\mathrm{KdV}$ possesses the famous sech ${ }^{2}$ solitary wave solution $u(x, t)=$ $u_{0} \operatorname{sech}^{2}\left((x-c t) \sqrt{u_{0} / \gamma} / 2\right)$, for $u_{0}=2\left(c-c_{0}\right) / \epsilon_{1}$ and $\gamma=3 \epsilon_{2} /\left(5 \epsilon_{1}\right)$, see [1].

The Benjamin-Bona-Mahoney equation (BBM),

$$
u_{t}+c_{0} u_{x}+\frac{3 \epsilon_{1}}{2} u u_{x}-\frac{3 \epsilon_{2}}{20} c_{0}^{-1} u_{x x t}=0
$$

has the same $\operatorname{sech}^{2}$ shape for its solitary wave, but with $\gamma$ replaced by $\gamma^{\prime}=c \gamma / c_{0}$. BBM is asymptotically equivalent to $\mathrm{KdV}$ at order $O\left(\epsilon_{1}, \epsilon_{2}\right)$. However, the linear dispersion relation for BBM is a better match than that for $\mathrm{KdV}$ in the physical application to shallow water waves.

Beyond $\mathrm{KdV}$ at linear order, the asymptotic expansion at quadratic order in the small parameters $\epsilon_{1}$ and $\epsilon_{2}$ does not produce a unique wave equation for unidirectional shallow water waves [2]. Instead, the asymptotic 
expansion produces an entire family of shallow water wave equations that are asymptotically equivalent to each other at quadratic order in the shallow water expansion parameters. The equations in this family are related amongst themselves by a continuous, three-parameter group of nonlinear, nonlocal transformations of variables given by,

$$
u=v+\epsilon_{1}\left(a_{1} v^{2}+a_{2} v_{x} \partial_{x}^{-1} v\right)+\epsilon_{2} a_{3} v_{x x},
$$

in which $\left(a_{1}, a_{2}, a_{3}\right)$ are real parameters. This transformation group was first introduced for determining normal forms of shallow water equations by Kodama in 3 .

Among the family of asymptotically equivalent shallow water wave equations at quadratic order accuracy in the small parameters $\epsilon_{1}=a / h$ and $\epsilon_{2}=h^{2} / l^{2}$ are several equations that are completely integrable. As for $\mathrm{KdV}$ at linear order, these integrable shallow water equations at quadratic order possess soliton solutions that interact via elastic collisions. In particular, the equation in the KdV hierarchy with fifth-order derivatives is present among these integrable equations, as shown in [4.

The family of shallow water equations at quadratic order accuracy that are asymptotically equivalent under Kodama transformations (3) contains the following sub-family, derived in [2],

$$
\underbrace{m_{t}}_{\text {Evolution }}+\underbrace{c_{0} u_{x}+\frac{3 \epsilon_{2}}{20} u_{x x x}}_{\text {Dispersion }}+\underbrace{\epsilon_{1}\left(u m_{x}+b m u_{x}\right)}_{\text {Nonlinearity }}=0
$$

where $m=u-\left(19 \epsilon_{2} / 60\right) u_{x x}$. The set of $1+1$ evolutionary equations (4) is nonlocal, dispersive and nonlinear. The nonlinearity in this set of asymptotically equivalent shallow water equations is parameterized by the real constant $b$, which depends on the group parameters $\left(a_{1}, a_{2}, a_{3}\right)$ in the Kodama transformation (3). An asymptotically equivalent shallow water equation for any $b \neq-1$ may be achieved by a Kodama transformation. However, the case $b=-1$ violates the asymptotic ordering and the corresponding Kodama transformation is singular in this case [2].

The cases $b=2$ and $b=3$ are special values for the $b$-equation (44). The case $b=2$ restricts (4) to the integrable Camassa-Holm equation (CH) [5]. The case $b=3$ in (4) is the Degasperis-Procesi equation (DP) [6], which was shown to be integrable in [7. These two cases exhaust the integrable candidates for (4), as was shown using Painlevé analysis in [7]. The b-family of equations (4) was also shown in [8] to admit the symmetry conditions necessary for integrability only in the cases $b=2$ for $\mathrm{CH}$ and $b=3$ for DP. 
$\mathrm{KdV}$ (11) and the cases $b=2(\mathrm{CH})$ and $b=3(\mathrm{DP})$ of equation (4) are three completely integrable Hamiltonian equations that possess solitons as traveling waves. In $\mathrm{KdV}$, the balance that confines the traveling wave soliton occurs between nonlinear steepening and linear dispersion. This is the leading order asymptotic balance for shallow water waves. However, even in the absence of linear dispersion, the parameter $b$ in equation (4) introduces additional possibilities for balance, including the nonlinear/nonlocal balance in the following (rescaled) dispersionless case of $\mathrm{CH}$ that was studied previously for $b=2$ in [5],

$$
m_{t}+u m_{x}+b m u_{x}=0, \text { with } m=u-\alpha^{2} u_{x x} .
$$

The nonlinear/nonlocal balance in this equation, even in the absence of linear dispersion, can still produce a confined solitary traveling wave pulse $u(x, t)=c e^{-|x-c t| / \alpha}$, called the peakon [5]. The peakon moves with speed equal to its amplitude and has a jump in derivative at its peak. Peakons for either $b=2$ or $b=3$ are true solitons that interact via elastic collisions under $\mathrm{CH}$ dynamics, or DP dynamics, respectively. In addition, the $\mathrm{CH}$ and DP initial value problems are both completely integrable as Hamiltonian systems by using the inverse spectral transform (IST) method for an isospectral linear eigenvalue problem whose purely discrete spectrum gives the asymptotic speeds of the peakons [5], 7]. Figure 1 shows the evolution under dispersionless $\mathrm{CH}$ for the case $b=2$ in equation (5) of a Gaussian initial velocity distribution of unit area and width $5 \alpha$. Peakon solutions exist for equation (5) with any value of $b$. However, we shall find that the stability of these peakon solutions requires $b>1$.

The properties of dispersionless $\mathrm{CH}$ for the case $b=2$ in equation (5) and also the related class of dispersionless equations consisting of (5) with $u=g * m=\int_{-\infty}^{\infty} g(x-y) m(y) d y$ for the case $b=2$ and an even kernel $g(x)=g(-x)$ including $g(x)=e^{-|x| / \alpha}$ are studied further in Fringer and Holm [9]. The properties of dispersionless DP for the case $b=3$ in equation (5) are studied further in [7, 10].

The dispersionless limit of KdV in (11), upon rescaling velocity $u$, yields the Burgers equation

$$
u_{t}+u u_{x}-\nu u_{x x}=0,
$$

in which we now also add constant viscosity $\nu$. The characteristic Burgers solution is the classic ramp and cliff shown arising from the Gaussian initial condition in Figure 2. In the ramp/cliff solution, nonlinear steepening is balanced by linear viscosity to produce the "cliff" whose width is controlled 
D. D. Holm \& M. F. Staley Solitons, peakons, ramps/cliffs and leftons 5

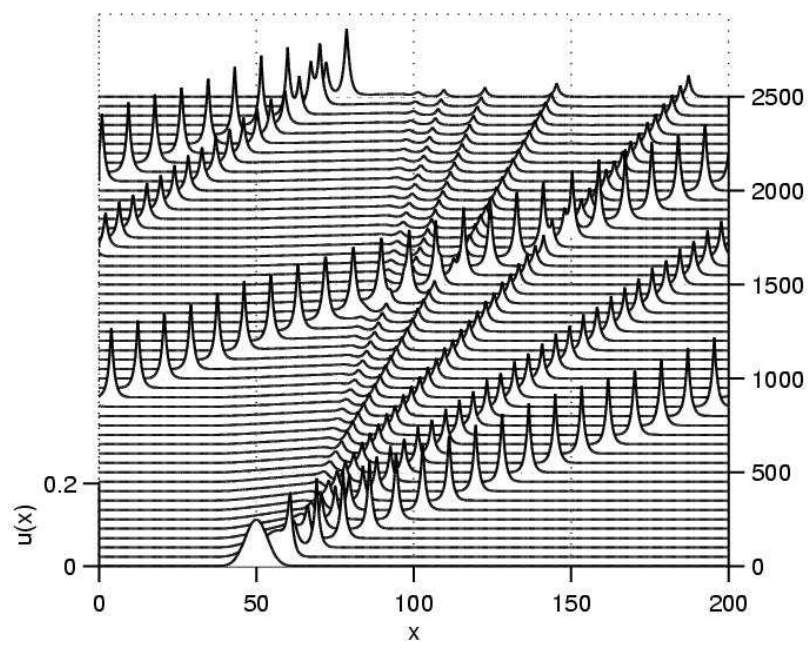

Figure 1: Evolution under equation (5) with $b=2$ of a Gaussian initial velocity distribution of unit area and width $5 \alpha$.

by the magnitude of viscosity $\nu$. The "ramp" is the self-similar $u \approx x / t$ part of the solution for which the viscous term vanishes.

The equations KdV, BBM, CH, DP, the other $b$-equations and Burgers all preserve the area $M=\int_{-\infty}^{\infty} u d x$ (total momentum) for a solution that vanishes at spatial infinity.

\subsection{Problem statement for the present work}

In this paper, we shall treat the constant $b$ in the dispersionless case of equation (4) as a bifurcation parameter. We shall study numerically the exchange of stability among three types of solitary wave solutions, as $b$ is varied. This is done by using numerical simulations to investigate its initial value problem on a periodic domain of length $L \gg \alpha$ for various values of 


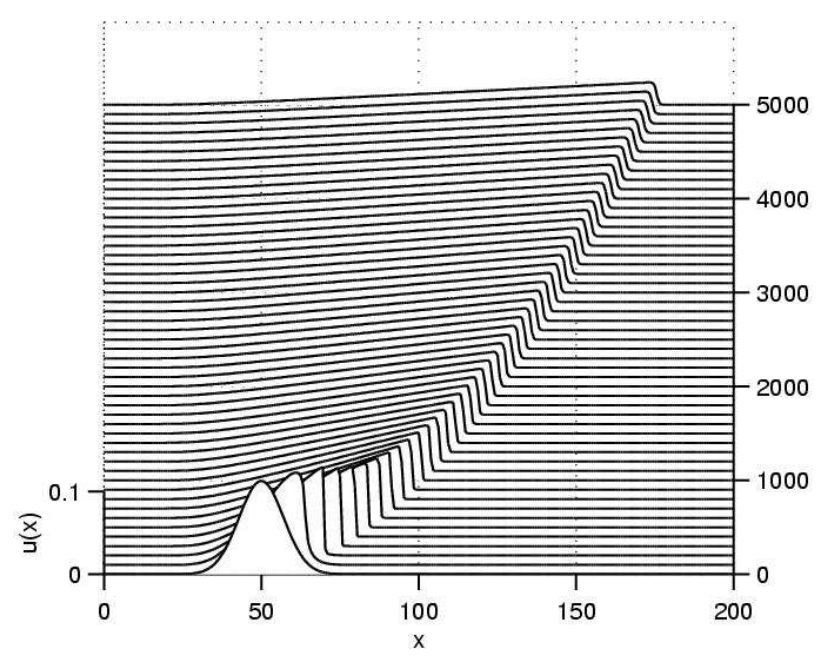

Figure 2: The Burgers solution is the classic ramp/cliff shown arising from the Gaussian initial condition.

the parameter $b$. When necessary, we shall also add viscosity in the form ${ }^{1}$

$$
m_{t}+\underbrace{u m_{x}}_{\text {convection }}+\underbrace{b u_{x} m}_{\text {stretching }}=\underbrace{\nu m_{x x}}_{\text {viscosity }}
$$

with $m=u-\alpha^{2} u_{x x}$. We shall treat the following cases, which we shall show analytically are special values: $b=0, \pm 1, \pm 2, \pm 3$.

\section{Exchange of stability}

\subsection{Numerics for exchange of stability from $b=0$ to $b= \pm 2$}

We begin by considering the initial value problem for case $b=0$ in equation (17). The first part of Figure [3 shows for $b=0$ that a peakon of width $w=5 \alpha$ is unstable to forming a ramp/cliff. Namely, the initial peakon develops into a Burgers-type ramp/cliff solution for $b=0$, just as it would

\footnotetext{
${ }^{1}$ Relation of the $b$-equation (4) to $3 \mathrm{D}$ turbulence closure models. Higherdimensional representatives of the viscous $b$-family of equations (4) have appeared recently in 3D turbulence modeling. The $b=0$ case of equation (7) is the $1 \mathrm{D}$ version of Leray's regularization of the Navier-Stokes equations in 3D [11. Leray regularization has recently been revived as an approach to deriving turbulence closure models for large eddy simulations of turbulent mixing layers in 3D [12. For 3D studies of the turbulence model corresponding to the $b$-equation (4) with $b=2$, see [13], 14, 15].
} 


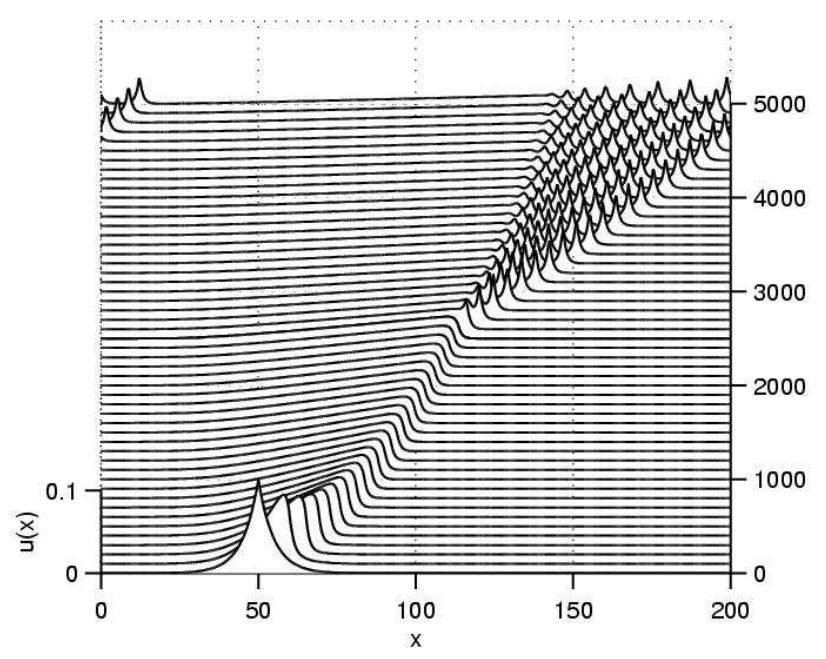

Figure 3: Exchange of stability between the classic ramp/cliff for $b=0$ and the peakons for $b=2$.

have done for the Burgers equation. Note, however, that the case $b=0$ in equation (17) is not the Burgers equation. At time $T=2500$, we stopped this part of the numerical calculation and used the result as the initial condition for continuing the $b$-equation evolution with $b$ changed to $b=2$, a case that supports peakons. The remainder of Figure 3 shows the exchange of stability after $b=0$ is changed to $b=2$. Thus, the Burgers-type ramp/cliff solution is stable for $b=0$ in equation (17). However, when treated as an initial condition, the ramp/cliff solution is unstable and breaks into a train of peakons for $b=2$. Figure 4 shows the spatial profiles of these solutions at the initial time, at the end of the $b=0$ evolution, and at the end of the $b=2$ evolution. A similar exchange of stability occurs between the ramp/cliff for $b=0$ and the train of peakons for $b=3$.

If we switch from $b=0$ to $b=-2$ instead of $b=2$, then Figure 5 shows that the ramp/cliff at time $T=2500$ evolves into a train of leftward moving solitary waves (leftons). Thus, a different exchange of stability occurs between ramp/cliff solutions for $b=0$ and leftons for $b=-2$ (and for $b=-3$ as well). Figure 6 plots the comparison between the stationary solution $u=u_{0} \operatorname{sech}^{2}(x /(2 \alpha))$ for $b=-2$ and the final states for an initial Gaussian of width $w=10$ evolved to leftons. Similar agreement occurs for the case $b=-3$, whose stationary solution is $u=u_{0} \operatorname{sech}(x / \alpha)$. Thus, confined initial states move leftward and evolve into stationary solutions for $b=-2$ and $b=-3$. 
D. D. Holm \& M. F. Staley Solitons, peakons, ramps/cliffs and leftons 8

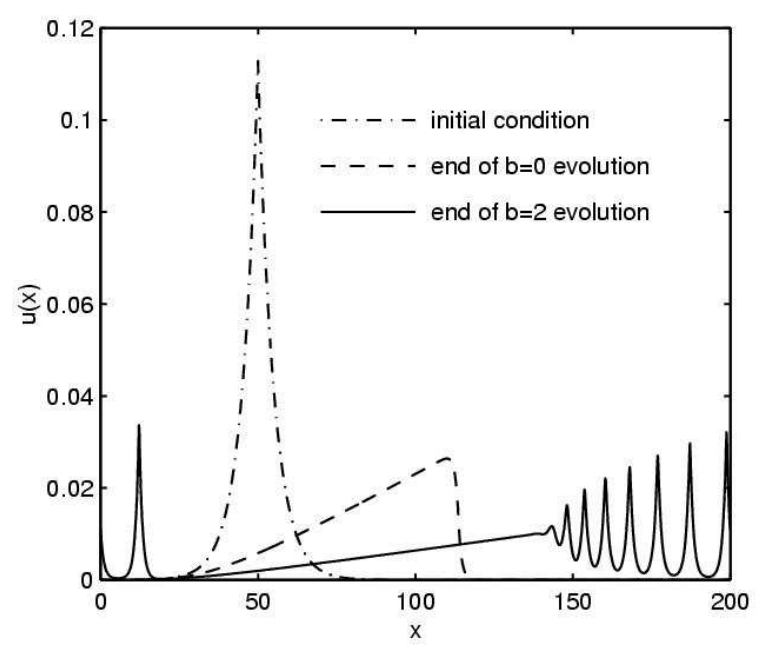

Figure 4: Spatial profiles of the solutions at the initial time and at the end times of the $b=0$ evolution and the $b=2$ evolution.

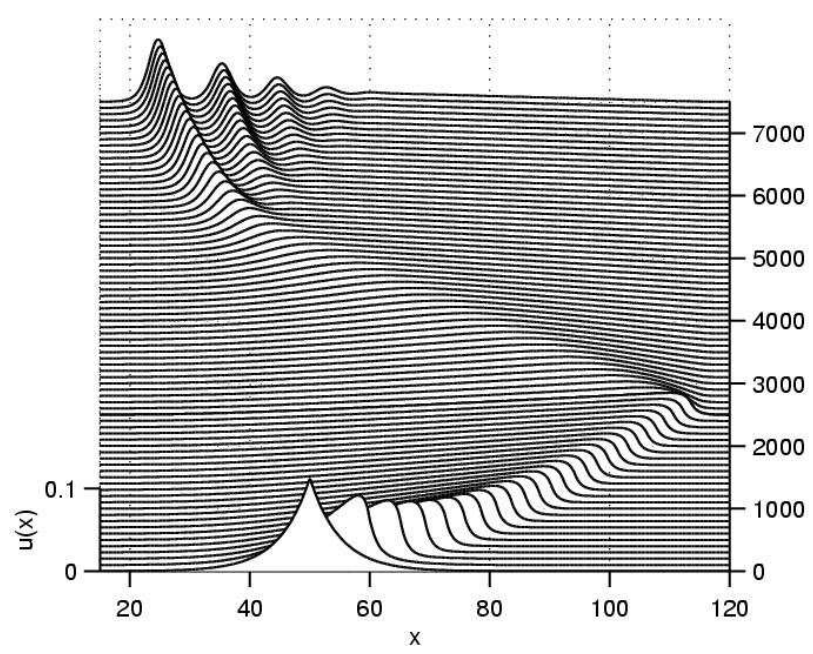

Figure 5: Exchange of stability between the classic ramp/cliff for $b=0$ and the leftons for $b=-2$. 
D. D. Holm \& M. F. Staley Solitons, peakons, ramps/cliffs and leftons 9

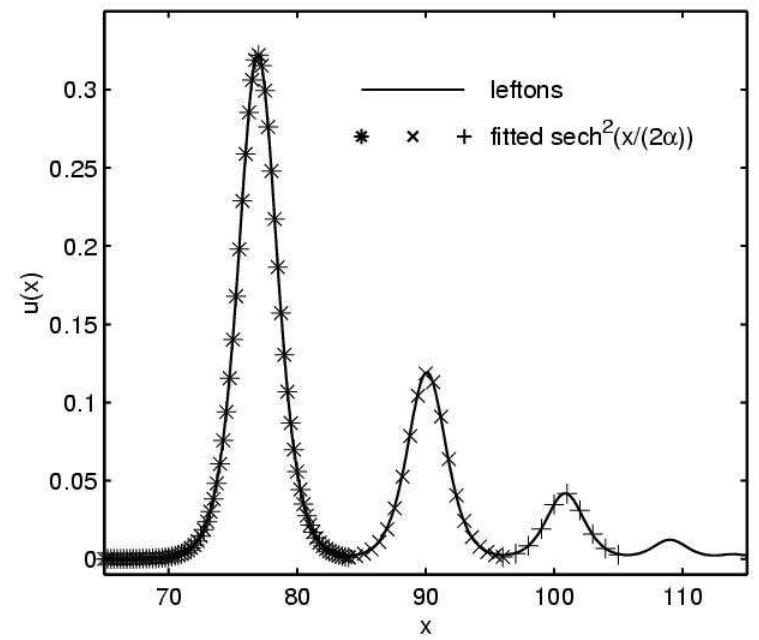

Figure 6: Comparison between leftons and the stationary solution $u=$ $u_{0} \operatorname{sech}^{2}(x /(2 \alpha))$ for $b=-2$.

\subsection{Analytical discussion of the exchange of stability}

The exchange of stability as the parameter $b$ varies can be understood analytically as arising from the following properties of the $b$-equation (7).

- For $0 \leq b \leq 1$, the $L^{1 / b}$ norm of the momentum $\|m\|_{L^{1 / b}}=\left(\int_{-\infty}^{\infty}|m|^{1 / b}\right)^{b}$ is preserved by (17), in the inviscid case for which $\nu=0$. Thus, the ramp/cliff solution for $b=0$ forms in a regime of classical analytical control in which $|m|$ possesses a maximum principle. Numerically, we observe: (1) the cliff's width is controlled by the magnitude of $\alpha$; and (2) the viscosity $\nu$ can be made negligible without measurably affecting the computed solution.

- For $1<b \leq 3$ in the inviscid case, an inflection point with negative slope may be shown to develop into a vertical slope in finite time. However, numerically, we observe the nonlinear evolution conspires to eliminate such singular inflection points, e.g., by forming peakons, which are weak solutions. - For $b=-1$ the lowest order nonlinearity in equation (77) (the steepening term $u u_{x}$ ) has coefficient zero. The evolution then develops from the higher order nonlinear terms. This can be seen by rewriting the $b$-equation (7) equivalently as

$$
u_{t}+(b+1) u u_{x}-\nu u_{x x}=\alpha^{2}\left(u_{t}+u u_{x}-\nu u_{x x}+\frac{b-3}{2} u_{x}^{2}\right)_{x x} .
$$

Thus, solutions with small curvature are nearly stationary for $b=-1$. The 
leading order nonlinearity is absent, so the dynamics is governed by the balance between viscous diffusion and higher-order terms. The case $\alpha \rightarrow \infty$ in (8) is interesting, as well. However, this case will be discussed elsewhere. - Equation (7) has stationary $(c=0)$ solutions $u=u_{0} \operatorname{sech}^{2}(x /(2 \alpha))$ for $b=-2$ and $u=u_{0} \operatorname{sech}(x / \alpha)$ for $b=-3$. Figure 6 shows that the evolution of equation (7) rapidly approaches the stationary solution for $b=-2$. (A similar result holds for $b=-3$.) The linearized evolution of (7) around these stationary solutions is stable for all wavelengths. Therefore, for any nonzero viscosity, these stationary solutions have a nontrivial basin of attraction. Note: equation (7) with either $b=-2$, or $b=-3$ is not Hamiltonian. The mathematical properties of the $b$-equation in these cases is discussed in [10].

\subsection{Confirmations of our numerical methods}

In our numerical runs we advanced the $b$-equation (7) with an explicit, variable timestep fourth/fifth order Runge-Kutta-Fehlberg (RKF45) predictor/corrector. We selected the timestep for numerical stability by trial and error, while our code selected the timestep for numerical accuracy (not to exceed the timestep for numerical stability). We used a very strict relative error tolerance per timestep, $\epsilon=10^{-8}$.

We computed spatial derivatives using 4th order finite differences, generally at resolutions of $2^{13}=8192$ or $2^{14}=16384$ zones. To invert the Helmholtz operator in determining $u(x, t)$ from $m(x, t)$, we used the Fourier transform. When the numerical approximation of the nonlinear terms had aliasing errors in the high wavenumbers, we removed these errors by applying a high pass filtered artificial viscosity.

The quality of the numerical convergence may be checked analytically in the collision of two peakons of asymptotic speeds $c_{1}$ and $c_{2}$, for which the minimum peakon separation with $b>1$ is

$$
e^{-\left|q_{\text {min }}\right| / \alpha}=1-\left(\frac{4 c_{1} c_{2}}{\left(c_{1}+c_{2}\right)^{2}}\right)^{1 /(b-1)} .
$$

For $b=2, c_{1}=1, c_{2}=1 / 2$ and $\alpha=5$, formula (9) implies $q_{\min }=$ $10 \ln 3=10.9861$. Our numerical results with the resolution of $2^{14}$ zones yield $q_{\min }=11.0049$. The small discrepancy, less than $0.2 \%$, occurs largely because our numerical measurement of $q_{\min }$ is obtained by examining the peakon positions at each internal timestep in the code without interpolation, although one is unlikely to land exactly on the time at which the minimum separation occurs. The code's true accuracy is better than the above measure indicates, because the intermediate steps involved in advancing the 
solution from one discrete time to the next with an RKF45 method cancel the higher-order discretization errors.

Likewise, for peakons with $b=3$, formula (9) gives the minimum separation when $c_{1}=1, c_{2}=1 / 2$, and $\alpha=5$, as $q_{\min }=5 \ln (3 /(3-\sqrt{8}))=14.3068$. In this case, our numerical results yield $q_{\min }=14.2924$, a discrepancy of only $0.1 \%$.

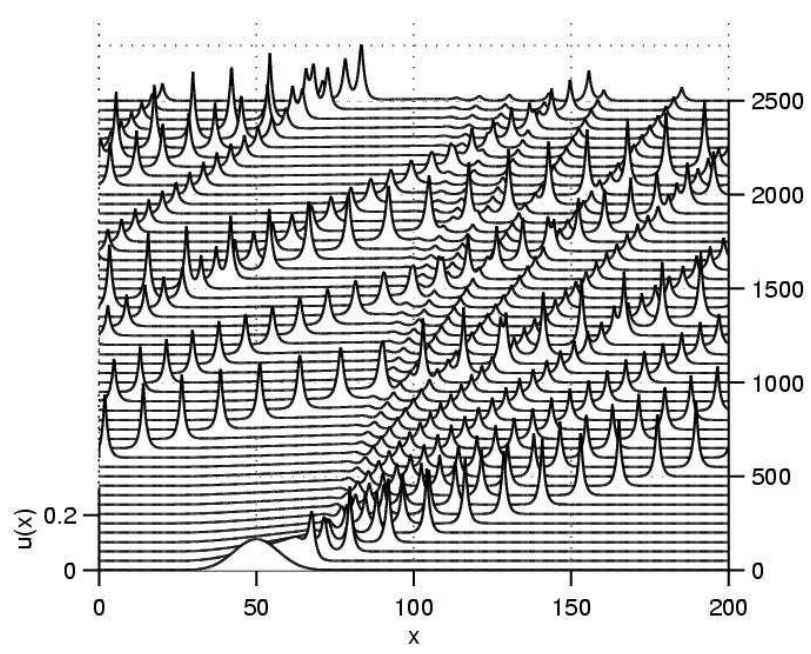

Figure 7: Scores of peakon collisions arise for $b=3$ (DP) starting from an initial Gaussian velocity distribution of width $w=10$.

Of course, the two-peakon collision is rather simple compared to the plethora of other multi-wave dynamics that occurs in this problem, as in Figures 7 and 8 For this reason, we also checked the convergence of our numerical algorithms by verifying that the relative phases of the peakons in the various figures remained invariant under grid refinement. Moreover, the integrity of the waveforms in our figures attests to the convergence of the numerical algorithm - after scores of collisions, the peakon waveforms are still extremely well preserved. The preservation of these peakon/soliton waveforms after so many collisions would not have occurred unless the numerics had converged well.

\section{Conclusions and further comments}

Equation (51) comprises a family of reversible, parity invariant, evolutionary $1+1$ PDEs that arise in the zero-dispersion case of shallow water waves at 
D. D. Holm \& M. F. Staley Solitons, peakons, ramps/cliffs and leftons 12

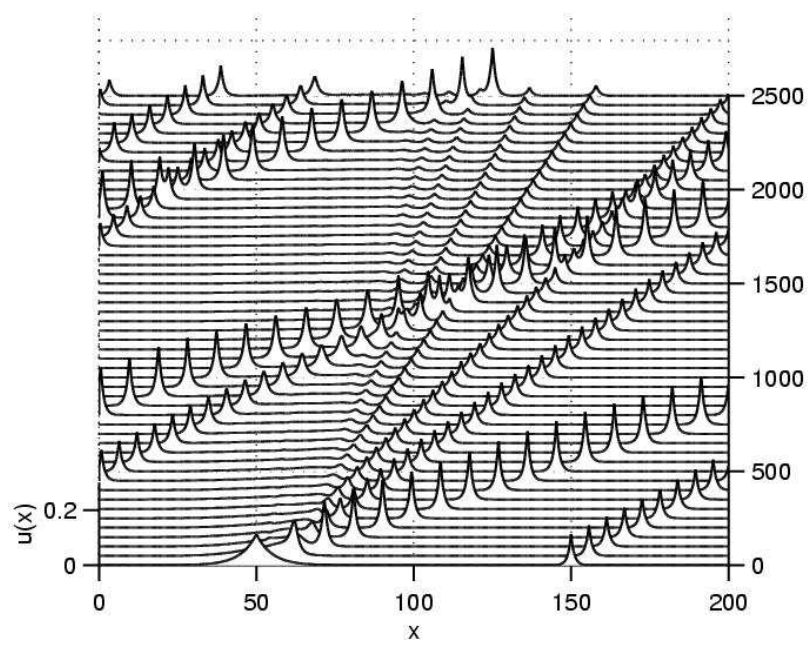

Figure 8: Under DP in (5) with $b=3$, many peakons of unit width for $\alpha=1$ emerge from an initial pair of peakons of width $w=5$.

quadratic order in the standard asymptotic expansion,

$$
m_{t}+u m_{x}+b m u_{x}=0, \text { with } m=u-\alpha^{2} u_{x x} .
$$

The paper identified the bifurcations and exchanges of stability of its traveling wave solutions as a function of the nonlinear balance parameter $b$.

Numerical computations of the initial value problem for (10) when $b>1$ showed the emergence of its stable particle-like peakon solutions and their interactions. These may be obtained analytically by superposing $N$ peakon traveling wave solutions for the inviscid case, $u(x, t)=c e^{-|x-c t|}$ as

$$
u(x, t)=\sum_{i=1}^{N} p_{i}(t) e^{-\left|x-q_{i}(t)\right|} \quad \text { and } \quad m(x, t)=\sum_{i=1}^{N} p_{i}(t) \delta\left(x-q_{i}(t)\right),
$$

for any real constant $b$. For any $b>1$, the peakons are stable and undergo particle-like dynamics in terms of the moduli variables $p_{i}(t)$ and $q_{i}(t)$, with $i=1, \ldots, N$. The peakon dynamics studied for $b>1$ in this framework displayed all of the classical soliton interaction behavior for peakons found in [5], 9] for the case $b=2$. This behavior included pairwise elastic scattering of peakons, dominance of the initial value problem by confined pulses and asymptotic sorting according to height - all without requiring complete integrability. Thus, the "emergent pattern" for $b>1$ in the nonlinear evolution governed by the $b$-equation (10) was the rightward moving peakon train, ordered by height. 
A second type of emergent pattern of the initial value problem for (10) occurs in the parameter region $0 \leq b<1$. This is the classic Burgerstype ramp/cliff structure as in Figure 4. In contrast, for the parameter region $b<-1$ a third type of behavior arises, consisting of leftward moving structures as in Figure 5. That the solution behavior should depend on the value of $b$ is clear from the velocity form of equation (10) written in (8).

Three regions of $b$. We found that the solution behavior for the $b$-equation (10) changes its character near the two boundaries, $b= \pm 1$, of the following three regions in the balance parameter $b$.

(B1) In the stable peakon region $b>1$, the Steepening Lemma for the $b$-equation (10) proven in [5] for $b=2$ and in [10] for $1<b \leq$ 3 allows inflection points with negative slopes to escape verticality by producing a jump in spatial derivative at the peak of a traveling wave that eliminates the inflection points altogether. Peakon behavior dominates this region. When $b \leq 1$ we found the solution behavior of the $b$-equation (10) changed its character and excluded the peakons entirely.

(B2) In the Burgers region $0 \leq b \leq 1$, the $L^{1 / b}$ norm of the variable $m$ is controlled $^{2}$ and the solution behavior is dominated by ramps and cliffs, as for the usual Burgers equation. Similar Burgers-type ramp/cliff solution properties hold for the region $-1 \leq b \leq 0$, for which the $L^{1 / b}$ norm of the variable $1 /|m|$ is controlled. At the boundary of the latter region, for $b=-1$, the active transport equation (10) admits stationary plane waves as exact nonlinear solutions. However, shallow water asymptotic ordering is broken at $b=-1$.

(B3) In the steady pulse region $b<-1$, pulse trains form that move leftward from a positive velocity initial condition (instead of moving rightward, as for $b>-1$ ). These leftward-moving pulse trains are found to approach a steady state.

At the two boundaries, $b= \pm 1$, of these three regions in $b$, the emergent solutions of the $b$-family of equations (10) are observed numerically to exchange stability. Although we discussed some analytical indications, a full explanation of these properties remains open for future research.

\footnotetext{
${ }^{2}$ For $b=0$, this is a maximum principle for $|m|$.
} 
D. D. Holm \& M. F. Staley Solitons, peakons, ramps/cliffs and leftons 14

\section{Acknowledgments}

We are grateful to A. Degasperis, A. N. W. Hone, J. M. Hyman, S. Kurien, C. D. Levermore, R. Lowrie and E. S. Titi for their thoughtful insights and remarks. This work was supported by the US DOE under contracts W-7405ENG-36 and the Applied Mathematical Sciences Program KC-07-01-01.

\section{References}

[1] M.J. Ablowitz and P.A. Clarkson, Solitons, Nonlinear Evolution Equations and Inverse Scattering, Cambridge University Press (1991).

[2] H.R. Dullin, D.D. Holm and G. Gottwald, Phys. Rev. Lett., 87, 194501 (2001).

[3] Y. Kodama, Phys. Lett. A 107, 245-249 (1985); Ibid 112, 193-196 (1985); Ibid 123, 276-282 (1987).

[4] Z. Li and N.R. Sibgatullin, J. Appl. Maths. Mechs. 61, 177-182 (1997).

[5] R. Camassa and D.D. Holm, Phys. Rev. Lett. 711661 (1993).

[6] A. Degasperis and M. Procesi, Asymptotic integrability, in Symmetry and Perturbation Theory, edited by A. Degasperis and G. Gaeta, World Scientific (1999) pp.23-37.

[7] A. Degasperis, D. D. Holm and A. N. W. Hone, A new integrable equation with peakon solutions. Theoret. and Math. Phys. 133, 1463-1474 (2002). http://xxx.lanl.gov/abs/nlin.SI/0205023.

[8] A.S. Mikhailov and V. Novikov, (private communication).

[9] O. Fringer and D.D. Holm, Physica D 150237 (2001).

[10] D.D. Holm and M.F. Staley, Wave structures and nonlinear balances in a family of $1+1$ evolutionary PDEs. To appear in SIAM J. Dyn. Syst. (2003).

[11] J. Leray, Sur le mouvement d'un liquide visqueux emplissant l'espace, Acta Math. 63, 193-248 (1934). Reviewed, e.g., in P. Constantin, C. Foias, B. Nicolaenko \& R. Temam, Integral manifolds and inertial manifolds for dissipative partial differential equations. Applied Mathematical Sciences, 70, (Springer-Verlag, New York-Berlin, 1989). 
D. D. Holm \& M. F. Staley Solitons, peakons, ramps/cliffs and leftons 15

[12] B.G. Geurts and D.D. Holm, Alpha-modeling strategy for LES of turbulent mixing. In Turbulent Flow Computation, edited by D. Drikakis and B. G. Geurts, Kluwer: London, pp. 237-278.

[13] S.Y. Chen, C. Foias, D.D. Holm, E.J. Olson, E.S. Titi and S. Wynne, Phys. Rev. Lett. 815338 (1998). Ibid, Physica D 13349 (1999). Ibid, Phys. Fluids 112343 (1999).

[14] S.Y. Chen, D.D. Holm, L.G. Margolin and R. Zhang, Physica D 133 66 (1999).

[15] C. Foias, D.D. Holm and E.S. Titi, Physica D 152505 (2001). Ibid, Dyn. and Diff. Eqns. 141 (2002). 